\title{
Editorial: Algorithmic requirements for HPC
}

\author{
Gabriel Wittum ${ }^{1}$
}

Published online: 8 September 2015

(C) Springer-Verlag Berlin Heidelberg 2015

The papers in this issue present work supported by DFG in the project ExaSolvers. The objective of the project is to develop optimal complexity solvers for exascale computing. The papers describe several facets of this development.

\section{The paradoxon of high performance computing}

Optimal complexity solvers are crucial to large scale computing. This can be seen from the following paradoxon of high performance computing.

Let an algorithm have execution time $E=O\left(n^{q}\right), q>1$. Let us further assume, that we buy a new computer, which is larger and faster by a factor of $\alpha>1$ than the old one. To compute a problem of size $\alpha \cdot n$ the new computer needs

$O\left(\alpha^{q} \cdot n^{q}\right)=\alpha^{(q-1)} \alpha E$,

meaning that the computing time increases by a factor of $\alpha^{(q-1)}>1$. Consequently, the larger the computer becomes, the longer the computing time will be. This is the paradoxon of high performance computing. It can be avoided only, if $q=1$, i.e. we use optimal order algorithms. Large scale computing makes sense only using algorithms with optimal complexity.

\section{The importance of adaptivity for high performance computing}

Adaptivity is a key tool for HPC, the larger the problem becomes, the more important adaptive grid resolution becomes. This can be seen from the following example computed by Arne Nägel, Sebastian Reiter and Andreas Vogel.

Take the skin permeation model from (Kreienbühl et al, this issue) and do a weak scaling of this problem with uniform refinement up to 65,536 cores (JuQueen) and compare with computing the same problem using adaptive refinement. Plotting this into one graph, we obtain.
Gabriel Wittum

cvs@g-csc.de

1 Frankfurt, Germany 


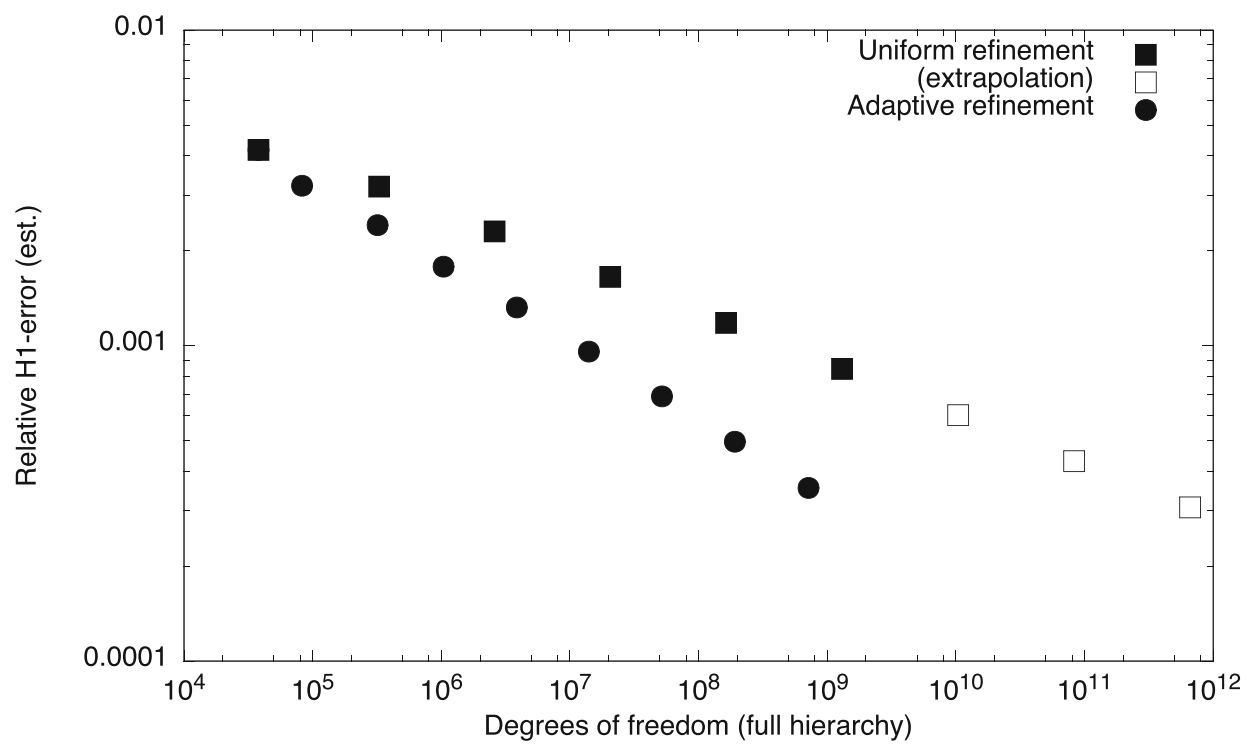

From that we conclude:

1. Using the full machine with the adaptive approach, we gain an accuracy comparable to the one with a computer 512 times as large.

2. Adaptivity is a key method for power saving. To reach the same error with the adaptive method, you need just 1,024 CPUs instead of 65,536 CPUs in the uniform case or using 65,536 CPUs for the adaptive computation, we would need a computer 512 times larger, i.e. with $33,554,432$ CPUs, to reach the same error on a uniform grid. This means saving $95.5 \%$ in CPU time and in power consumption.

3. Adaptivity gives a higher-order like effect, without requiring additional smoothness. This is due to the fact that in the uniform case, the number of degrees of freedom $N$ grows by a factor of 8 , while it grows by a factor of 3.7 only in the adaptive case.

4. This means, that the importance of adaptivity increases with growing system size, due to the different asymptotics.

5. Multi-scale modeling becomes mandatory for $H P C$. The smallest element in the uniform case is $25.9 \mathrm{~nm}$, in the adpative case, it is $2.7 \mathrm{~nm}$. Since the molecules making up the lipid bilayers are about $6 \mathrm{~nm}$ long, we resolve the molecular fine structure of the porous medium matrix. Thus we need to incorporate this into our models.

Again we see: It is worth while bringing sophisticated algorithms to the parallel machine. These algorithms enable qualitatively and quantitatively superior results, in particular w.r.t. the asymptotic behaviour. The larger the system to be solved and the corresponding computer is, the more important the choice of the algorithm becomes. Even though adaptive methods need more sophisticated software which perform slower per core, in our case by a factor of 4 . the gain of a factor of 512 shows the superiority of the adaptive approach. 